\title{
Animal Models of Neurologic Disorders: A Nonhuman Primate Model of Spinal Cord Injury
}

\author{
Yvette S. Nout • Ephron S. Rosenzweig • \\ John H. Brock • Sarah C. Strand • Rod Moseanko • \\ Stephanie Hawbecker • Sharon Zdunowski • \\ Jessica L. Nielson • Roland R. Roy • Gregoire Courtine • \\ Adam R. Ferguson • V. Reggie Edgerton • \\ Michael S. Beattie • Jacqueline C. Bresnahan • \\ Mark H. Tuszynski
}

\begin{abstract}
Primates are an important and unique animal resource. We have developed a nonhuman primate model of spinal cord injury (SCI) to expand our knowledge of normal primate motor function, to assess the impact of disease and injury on sensory and motor function, and to test candidate therapies before they are applied to human patients. The lesion model consists of a lateral spinal cord hemisection at the $\mathrm{C} 7$ spinal level with subsequent examination of behavioral, electrophysiological, and anatomical outcomes. Results to date have revealed significant neuroanatomical and functional differences between rodents and
\end{abstract}

\section{Y. S. Nout $(\bowtie)$}

Department of Animal and Veterinary Sciences, College of Agriculture, California State Polytechnic University,

Pomona, CA, USA

e-mail: ysnout@csupomona.edu

\author{
E. S. Rosenzweig • J. H. Brock • M. H. Tuszynski $(\bowtie)$ \\ Department of Neurosciences, University of California, \\ La Jolla, \\ San Diego, CA, USA \\ e-mail: mtuszynski@ucsd.edu
}

S. C. Strand $\cdot$ R. Moseanko $\cdot$ S. Hawbecker

California National Primate Research Center,

University of California,

Davis, CA, USA

S. Zdunowski $\cdot$ R. R. Roy $\cdot$ V. R. Edgerton

Department of Integrative Biology and Physiology,

Los Angeles, CA, USA

\section{R. Edgerton}

Departments of Neurobiology and Neurosurgery,

Los Angeles, CA, USA primates that impact the development of candidate therapies. Moreover, these findings suggest the importance of testing some therapeutic approaches in nonhuman primates prior to the use of invasive approaches in human clinical trials. Our primate model is intended to: 1) lend greater positive predictive value to human translatable therapies, 2) develop appropriate methods for human translation, 3) lead to basic discoveries that might not be identified in rodent models and are relevant to human translation, and 4) identify new avenues of basic research to "reverse-translate" important questions back to rodent models.

\section{S. Zdunowski $\cdot$ R. R. Roy • V. R. Edgerton Brain Research Institute, University of California, Los Angeles, CA, USA}

J. L. Nielson · A. R. Ferguson · M. S. Beattie · J. C. Bresnahan Brain and Spinal Injury Center, Department of Neurological Surgery, University of California,

San Francisco, CA, USA

G. Courtine

Experimental Neurorehabilitation, Department of Neurology, Universität Zurich,

Zurich, Switzerland

M. H. Tuszynski

Veterans Administration Medical Center,

La Jolla, CA, USA 
Keywords Hemisection - Cervical Spinal Cord Injury . Brown-Sequard · Monkey · Translational Medicine

\section{Introduction}

Animal models of disease are critical to improving the care of humans. Research using animal models increases our knowledge and understanding of disease mechanisms and evolution of injury, and provides a clinically relevant platform for developing and evaluating therapies. The eventual goal is to have successful discovery and preclinical testing of therapies that can be taken to human clinical trials. The primate model of spinal cord injury (SCI) described in this review has been developed during the last 10 to 15 years to establish a reliable means of preclinical assessment of candidate therapies for restoring locomotor function and skilled hand use after SCI, and for evaluating delivery methods and the safety of candidate therapies.

Before testing therapies in a human clinical trial, 1 requirement is demonstration of sufficient supporting preclinical data, usually obtained from animal models, to justify the effort, risk, and expense associated with human clinical trials [1]. For example, the Food and Drug Administration requires safety testing of potential therapeutics in animal models. Efficacy data from animal studies are not required, but the data are usually expected to accompany an investigational new drug application. Animal studies are also important for assessing the risk-to-benefit ratio of a proposed therapy. If the proposed treatment presents little to no risk (e.g., use of an established and safe medication for another disease indication), fewer animal studies are required prior to clinical trials. On the other hand, if the potential risk of a proposed therapy is higher (e.g., surgical manipulation of the lesion site and/or surrounding spared tissue in SCI), evidence of greater potential efficacy is necessary. It is for the latter studies in particular that a primate model of SCI can be beneficial [2].

Often there are multiple animal models to study 1 disorder. The most suitable model is selected based on its unique characteristics and ability to address the scientific questions of interest. There is no consensus among investigators regarding which of the many animal models of SCI best predicts potential beneficial outcome in humans [3]. Most commonly, rats or mice are used, and SCI is produced by transecting all or part of the spinal cord (e.g., dorsal or lateral hemisection, or complete transection), or by compressing or contusing the spinal cord in a controlled fashion (e.g., using a modified aneurysm clip or weight-drop device). We perform a lateral hemisection of the spinal cord, both for the precise control of lesion location and extent, and for the humane preservation of function it affords the experimental animals (see as follows).

An injury can be placed at a specific spinal level to generate a particular type of functional deficit that is the target of treatment. Traditionally, most rat studies have placed SCI contusions at the mid-thoracic vertebral level to produce bilateral hindlimb paralysis, a symptom seen in most humans after SCI. However, a recent survey has shown that the number of cervical vertebral level injuries is increasing [4], and regaining arm and hand function is considered the top priority for improving quality of life of quadriplegics [5]. Consequently, we, and others, are now placing unilateral injuries at the cervical level (C5 vertebral level) to better model human injury. Advantages to using cervical SCI models are: 1) clinical relevance: most human SCI injuries occur in the cervical area (53\%) [6], thus there is a large interest in recovery of hand function; 2) anatomy: proximity of cervical motor nuclei to the lesion means that even short distance axonal growth could improve forelimb function; 3 ) comparative anatomy and behavior: animal forelimb function can be compared to human arm/hand function; and 4) animal care and ethical considerations: in the rat, a unilateral cervical contusion injury primarily affects the ipsilateral forelimb [7] and results in minimal contralateral forelimb or hindlimb deficits, autonomic dysfunction (including the bowel and bladder dysfunction), or requirement for food or fluid support.

Rodent contusion models appear to replicate the mechanism of the majority of human SCIs. However, whether or not results obtained from studies in these models can be extrapolated to primates, including humans, depends on the specific motor task and the similarities in the anatomical and functional organization of the sensorimotor systems between lower and higher order mammals [2, 8, 9]. A good understanding of the comparative function of the sensorimotor system is critical to determining these limitations and understanding the relevance of the information that the different animal models provide. For example, there are distinct differences between rodent and primate spinal cord systems including:

1. Size: Regeneration of an injured axon in the rat for several millimeters might be sufficient to generate functional recovery, whereas distances of many centimeters might be required in the primate spinal cord. The difference in scale between the human and rodent cord is also a significant issue when investigating feasibility and effects of surgically applied therapies

2. Anatomy: There are differences between rodents and primates in the number, location, and termination patterns of important axonal systems, such as the corticospinal tract. These differences may contribute 
to altered responses to injury. For example, activation of stepping-related spinal circuitry may depend more on supraspinal input in nonhuman primates than in lower order mammals $[10,11]$. In addition, there are many more direct projections from the cortex to spinal motor neurons in primates than in lower order mammals [12-14].

3. Function: Some important axon systems have different functional roles in primates and rodents. For example, the corticospinal tract is critical for fine motor control in humans and nonhuman primates, but less critical in rats $[2,13,15]$. On the other hand, the rubrospinal tract is important for forelimb movement in rats, but is vestigial in humans [16].

4. Inflammatory Responses and Secondary Injury: Although not well-characterized, the intensity and nature of inflammation and secondary damage may differ in rodent and primates species due to differences in immune complexity and molecular (e.g., cytokine) recruitment to injury sites $[1,8]$.

This article describes a nonhuman primate spinal cord hemisection model in which we study anatomical questions related to spared axonal sprouting, translesional regeneration, and recovery of hand and locomotor function. To date, this nonhuman primate model of SCI has demonstrated significant differences in neuroanatomy between the rat and primate [14, 17], and in endogenous responses and axonal sprouting between the rat and primate [18]. In addition, this model has been instrumental in developing and testing translational treatment methodologies, including the observation that treatment methods used in rodents [19-26] were problematic in primates [27]. We believe that primates should be used sparingly and only when models in less developed species are inadequate for addressing important mechanistic or translational questions; there is a clear and necessary role for nonhuman primate studies under these conditions $[2,15]$.

\section{Model Description}

The University of California (UC) Spinal Cord Injury Consortium is a collaborative project among 5 campuses (UC San Diego, UC Los Angeles, UC Irvine, UC Davis, and UC San Francisco), which bring together the expertise in the fields of neuroanatomy, functional assessment, electrophysiology, growth factor gene delivery, rehabilitation after SCI, and primate care. The goal of this research program was to examine injury, plasticity, and regeneration in the injured adult primate spinal cord so that potential therapies could be translated to humans in a rational and efficient manner. The work of this consortium focused on enhancing mechanistic understanding of injury to the primate spinal cord, and developing practical and validated therapies that could undergo human testing in the future.

In the nonhuman primate model described in this review, adult male (6-10 years) Rhesus macaque monkeys (Macaca mulatta) were used. The animals were housed individually in cages in an Association for Assessment and Accreditation of Laboratory Animal Care (AAALAC), which is an approved animal facility at the California National Primate Research Center at UC Davis (CA). A 12-h day/night cycle was maintained. Water was freely available, and food was provided according to California National Primate Research Center guidelines. Enrichment (mirrors, forage boards, chew toys, and radio) were provided daily. In addition, animals received puzzle toys and food reinforcements (produce items and treats), and access to a large exercise enclosure 5 times per week. All behavioral testing was conducted in the same room where the animals were housed.

\section{SCI}

Our objective was to develop a nonhuman primate model of SCI that will permit assessment of candidate therapies for restoring both locomotor function and skilled hand use after SCI. In primates, many studies have evaluated functional recovery after spinal cord transection [28-31], mostly using lesions of specific funiculi to determine the role of ascending [32-35] and descending tracts, especially the corticospinal tract $[12,13,36-40]$. In initial studies we used a low thoracic (T10) hemisection to study the effect of a unilateral corticospinal tract lesion on quadrupedal stepping and recovery of fine motor control of the hindlimbs using tasks that required dexterous foot digit movements [41, 42]. Because the most common site of human SCI is the cervical spinal cord, and sprouting or regeneration of axons for short distances beyond a cervical lesion could restore hand function, we continued model development using a C7 (spinal cord level) lateral hemisection SCI.

To create a complete hemisection at the $\mathrm{C} 7$ spinal cord segment, the dorsal laminae were removed from the C6 vertebra and the caudal half of the C5 vertebra. This exposed the $\mathrm{C} 7$ spinal cord segment after which the dura was opened. A microblade mounted on a stereotaxic carrier was placed on the dorsal surface of the spinal cord $(0.5 \mathrm{~mm}$ contralateral) to the left of midline (i.e, positioned to make a slight over-hemisection). The blade was lowered through the cord to the vertebral canal floor, and then moved laterally to transect the right side of the spinal cord. The intradural right-sided hemisection was completed using microscissors under direct visual guidance of a high resolution intraoperative microscope to ensure total removal of any white matter in the lateral and ventromedial areas of the spinal cord. 


\section{Animal Care}

All surgeries were performed aseptically. Antibiotic therapy with cefazolin $(20 \mathrm{mg} / \mathrm{kg}$, i.m., three times a day) or cephalexin $(30 \mathrm{mg} / \mathrm{kg}$, orally, twice a day) was initiated preoperatively and continued for at least 7 days postoperatively. Food access was restricted for approximately $8 \mathrm{~h}$ preoperatively. Anesthesia was induced with ketamine $\mathrm{HCl}$ $(10 \mathrm{mg} / \mathrm{kg}, \mathrm{i} . \mathrm{m}$.) and maintained with isoflurane gas $(2 \%)$ in $100 \%$ oxygen. Atropine sulfate $(0.4 \mathrm{mg} / \mathrm{kg}, \mathrm{i} . \mathrm{m}$. $)$ was administered during induction. Heart rate, blood pressure, oxygen saturation, end-tidal $\mathrm{CO}_{2}$, body temperature, respiratory rate, respiratory pressure, and tidal volume were monitored throughout the surgery. Lactated Ringer's solution $(10 \mathrm{ml} / \mathrm{kg} / \mathrm{h})$ was administered for the duration of anesthesia. Analgesia was provided by either oxymorphone $(0.15 \mathrm{mg} / \mathrm{kg}$, i.m., three times a day) or buprenorphine $(0.01-0.03 \mathrm{mg} / \mathrm{kg}$, i.m., three times a day) and was continued postoperatively for a minimum of 3 days.

To date, all animals survived the surgical procedures, and most were released from the hospital within a few days of surgery. Detailed medical records were maintained while the animal was in the hospital and throughout the remainder of the experiment. Animals were returned to their home cages that were equipped with fleece pads and porous rubber mats to prevent pressure sores. Monkeys were attended to every 2 to $4 \mathrm{~h}$ during the day, and were encouraged with preferred food items, to sit up, reach, and stand. Skin lesions were treated, and if necessary limbs were bandaged. Animals that displayed self-injury may have received gabapentin (15$60 \mathrm{mg} / \mathrm{kg} /$ day $)$ and $/$ or haloperidol (0.01-0.05 mg/kg/day) in an effort to prevent further skin damage. In general, animals recovered the ability to sit and mobilize quickly; by 4 days post-hemisection, $90 \%$ of the animals are able to sit unassisted, and by 7 days, $65 \%$ of the animals were able to sit more than $50 \%$ of the time [43]. Development of spasticity was monitored by assessing the right hand and arm using the Modified Ashworth Scale [44] while the animal was seated in a testing chair prior to initiation of functional training. In our most recent study, progressive development of moderate spasticity was observed in approximately 4 of 20 monkeys, evolving for 3 to 6 months after hemisection.

\section{Outcomes: Function and Electrophysiology}

Locomotor and skilled hand function were evaluated when monkeys were walking on a treadmill, performing hand tasks in a restraining chair, and spontaneously moving in a large enclosed, yet open field [43]. Animals were trained, and baseline data was collected starting at 3 to 5 months before spinal cord hemisection. Details of these functional outcome tests have been recently published [43]. Briefly, after spinal cord hemisection, monkeys spent approximately 30 minutes 5 times per week in the open field, approximately 20 minutes 2 times per week on the treadmill (speeds of $0.45,0.89,1.34$, and $1.79 \mathrm{~ms}^{-1}$ ), and 30 minutes 3 times per week in the restraining chair. Observational scoring of the animal's activity and the ability to complete tasks (such as climbing, retrieving objects out of cups, and manipulating large objects) in the open field were scored once per week. Completion of skilled hand tasks in the restraining chair (e.g., retrieving small items from a platform, retrieving a grape from a stick, and pulling a handle) were scored 3 times per week. Kinematics and electrophysiological data were collected periodically from treadmill training and in the testing chair (baseline, 6, 9, 12, 16, 20, and 24 weeks postoperatively), and approximately once per month in the open field. For electrophysiological studies, bipolar intramuscular electromyography (EMG) electrodes (Konigsberg Instruments Inc., Pasadena, CA) were implanted into selected muscles for telemetric recording at 1 to 5 months prior to spinal cord hemisection [41, 42]. For kinematics analyses, reflective markers were attached bilaterally to the shaved skin overlying the greater trochanter, the knee joint, the malleolus, the fifth metatarsal, and the outside tip of the fifth digit, the head of the humerus, the elbow joint, the distal head of the ulna, the metacarpophalangeal joint, and the outside tip of the third digit. SIMI motion capture software (SIMI Reality Motion Systems, Unterschleissheim, Germany) was used to obtain threedimensional coordinates of the markers synchronized with EMG output [41, 42, 45].

These functional testing paradigms were developed for testing treatment efficacy in this primate model of SCI, and will also be useful for understanding the range of functional outcomes possible after spinal lesions in higher primates and humans. We recently showed that the range of recovery observed after removal of all ascending and descending fibers on 1 side of the spinal cord is substantially less (i.e., there is less variability in post-lesion performance between subjects) than observed after subtotal hemisection lesions [43]. Nonetheless, some animals recover substantially after a complete hemisection. To date, we have studied approximately 45 monkeys on hand and treadmill tasks, and approximately 25 monkeys in the open field. All animals show gradual improvement in function beginning approximately 2 weeks after hemisection, with the greatest rate of improvement between 3 and 8 weeks post-lesion. Overall, animals recover to approximately $50 \%$ of pre-hemisection baseline performance after a C7 hemisection. Detailed reports on recovery of function in this model have been published (Table 1) [18, 43].

After SCI in most animals, the ipsilesional hindlimb initially exhibits flaccid paralysis with the limb in extension, followed by gradual return of hip, knee, and ankle flexion. Most animals also rapidly recover extensive shoulder and 
Table 1 Recovery of Function with Time in the Open-Field Exercise Enclosure and On Hand Tasks of Rhesus Macaques after C7 Spinal Cord Hemisection

\begin{tabular}{|c|c|c|c|c|}
\hline $\begin{array}{l}\text { Week } \\
\text { Post SCI }\end{array}$ & Home cage & Exercise enclosure & Reaching tasks & Handle Pull \\
\hline 1 & $\begin{array}{l}\text { Initially recumbent. } \\
\text { By day } 4,90 \% \\
\text { of animals sit } \\
\text { unassisted }\end{array}$ & N/A & N/A & N/A \\
\hline $2-3$ & $\begin{array}{l}\text { Most animals sit } \\
\text { up }>50 \% \text { of time. }\end{array}$ & $\begin{array}{l}\text { Sitting and scooting; some } \\
\text { animals are able to support } \\
\text { weight and walk. }\end{array}$ & $\begin{array}{l}\text { Animals attempt to perform tasks } \\
\text { with the impaired right arm and } \\
\text { hand. Performance includes } \\
\text { reaching, touching, grasping, and } \\
\text { transferring food to the mouth. }\end{array}$ & $\begin{array}{l}\text { Animals attempt to complete } \\
\text { task. Gradual improvement } \\
\text { occurs from } 2-15 \text { weeks } \\
\text { after SCI. Animals perform } \\
\text { best on this task (recover } \\
\sim 70 \% \text { of pre-lesion function). }\end{array}$ \\
\hline $4-5$ & $\begin{array}{l}\text { General activity level } \\
\text { (sitting up and } \\
\text { moving around cage) } \\
\text { comparable to } \\
\text { pre-injury. }\end{array}$ & $\begin{array}{l}\text { All animals recover the ability } \\
\text { to stand and most }(60 \%) \\
\text { regain the ability to climb. } \\
\text { Sharpest rise in performance } \\
\text { occurs 3-8 weeks after SCI. }\end{array}$ & $\begin{array}{l}\text { Greatest rise in performance } \\
\text { on reaching tasks. Animals } \\
\text { often use alternative methods } \\
\text { to retrieve food (e.g., use of } \\
\text { hand like a rake rather } \\
\text { than pincer grasp) }\end{array}$ & \\
\hline $6-15$ & & $\begin{array}{l}\text { Further recovery of limb use } \\
\text { for walking and climbing, and } \\
30 \% \text { of animals use all } 4 \text { limbs, } \\
45 \% \text { use only } 3 \text { limbs, and } 25 \% \\
\text { use only the } 2 \text { intact side } \\
\text { limbs for movements in } \\
\text { the exercise enclosure }\end{array}$ & $\begin{array}{l}\text { Stabilization of performance } \\
\text { on reaching tasks with group } \\
\text { mean performance } \sim 50 \% \\
\text { of pre-lesion performance }\end{array}$ & \\
\hline $16-24$ & & $\begin{array}{l}\text { Performance of tasks in the } \\
\text { exercise enclosure stabilizes }\end{array}$ & & $\begin{array}{l}\text { Performance stabilizes. Few } \\
\text { animals are able to pull the } \\
\text { most heavily loaded springs }\end{array}$ \\
\hline
\end{tabular}

$\mathrm{N} / \mathrm{A}=$ not applicable; $\mathrm{SCI}=$ spinal cord injury

elbow use and develop limb use for weight support and stepping. When observed in the open field during the first 2 to 8 weeks after hemisection, animals rapidly recover sitting, standing, and ambulating, and most animals recover the ability to climb (Fig. 1). However, limb recruitment patterns vary widely. Ultimately, most animals use either 3 or 4 limbs for over the ground locomotion, whereas a small proportion of animals continue to use only the 2 unaffected limbs. Animals that do not regain weight support in the ipsilateral limbs either: 1) develop complete nonuse of the $\operatorname{limb}(\mathrm{s})$, or 2) suspend the $\operatorname{limb}(\mathrm{s})$ during locomotion, but still use the limb (typically poorly) for object support during food manipulation (forelimb) or for support during climbing (hindlimb). Typically, animals that regain good use of their impaired hand for object manipulation also use that hand for weight-supported stepping. In the hindlimb, abnormal placement of digit (D) 5 occurs consistently following SCI; D5 is placed adjacent to D4 with no spread visible, or slightly underneath $\mathrm{D} 4$, even in animals with good locomotor recovery.

Distal forelimb position and use varies after hemisection. Some animals regain little use of the wrist and digits, developing either wrist and finger joint extension or flexion

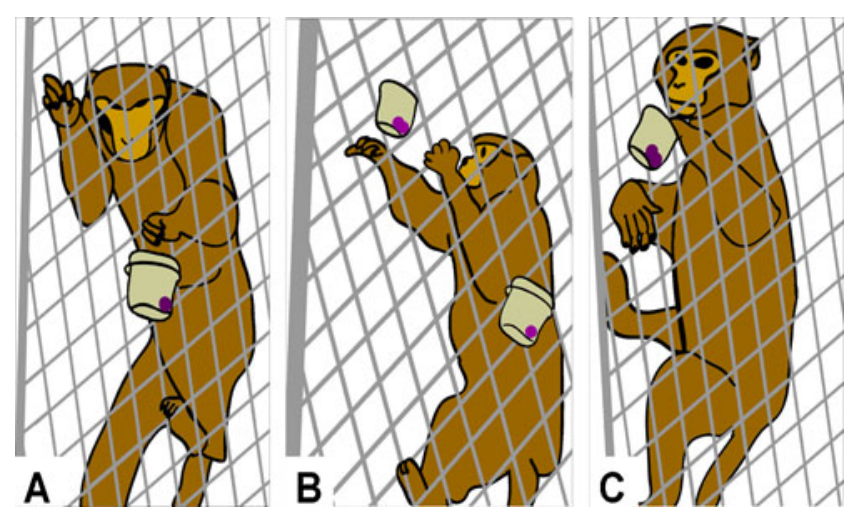

Fig. 1 Recovery of the ability to climb and retrieve food in the open field. (A) Schematic of a monkey in the open field before spinal cord injury (SCI) while the animal climbs up a fence and retrieves preferred food items from a cup that is hanging along the fence. The animal stabilizes itself along the fence by grasping the fence with its toes and fingers of 1 forelimb and both hindlimbs while they grasp the food from the cups with the hand of the second forelimb. (B, C) Schematics of a monkey in the open field at 6 months after SCI. These schematics depict an animal that did not regain the ability to use his fingers to grasp the fence, however, the animal was able to stick his hand through the fence (B) and flex his carpus/wrist around the fence to stabilize itself $(\mathbf{C})$. He then retrieved food from the cup with his left $(\mathbf{C})$ uninjured hand 
(clubbed hand), whereas a few regain nearly normal mobility and function. Poorly recovered animals have no or limited use of the ipsilesional hand. In animals with more normal use of the hand, there is more mobility in the wrist and digits, and they are able to partially extend the digits, open the hand, and apply a more normal grasp during manipulation of large objects. Similar to open field recovery, skilled hand task performance in the restraining chair gradually improves after hemisection, with the sharpest rise in performance at 4 to 5 weeks. Uninjured animals typically use a pincer grasp to pick up small food items, but after SCI, monkeys develop other retrieval strategies, including the use of the hand as a rake to get food from the platform (trapping the food between either the fingers or the fingers and the palm), and grasping food from the stick between the fingers and the palm with the hand rotated vertically (thumb up) and then lifting (Fig. 2). Of the skilled hand tasks, performance on the spring-loaded handle pull task is best [43] and continues to improve for a longer period of time ( $\sim 15$ weeks). This task requires the least dexterous movement of the hand.

Kinematics and EMG evaluations demonstrate failure to recruit most forelimb and hindlimb muscles on the affected side immediately after injury. This is followed by a period in which forelimb muscles exhibit substantial spontaneous levels of EMG activity without any hand use [18]. Fine motor control begins to recover at 4 to 8 weeks after injury, at which time EMG can (in some animals) show substantial, well-timed recruitment of forelimb muscles, including motor pools innervating the digit muscles. Coordinated patterns of muscle recruitment associated with successful food retrievals increase steadily, and usually reach a plateau (60\% of pre-lesion [18]) 12 to 16 weeks after injury. The ability to use the right forelimb and hindlimb during locomotion on a treadmill begins to recover at the same time as fine motor control (4-8 weeks post-lesion). Gait analysis at the onset of recovery in injured animals shows an inability to plantar step, limited weight bearing, and dragging the hind foot during the swing phase (Fig. 3). At 4 to 8 weeks after injury, there is substantial abnormal co-activation of extensor and flexor motor pools innervating the distal forelimb muscles, followed by steady improvement of locomotor function. Recovery of the ability to reciprocally recruit extensor and flexor digit muscles allows injured animals to regain limited movements in the distal forelimb extremities. The EMG amplitude and temporal patterns, however, remain significantly different from intact animals. A strong correlation exists between recovery of locomotion and fine motor control capacities (as assessed by kinematics and EMG). In addition, animals that recover extensive forelimb use during stepping also regain the highest levels of skilled hand function during food retrieval tasks [43]. Developing methods to evaluate behavioral recovery is ongoing and requires expertise and techniques spanning multiple fields, including neuroanatomy, electrophysiology, primate behavior and locomotion, and primate rehabilitation.

\section{Outcomes: Anatomical}

Most animals undergo anterograde labeling of the corticospinal tract 6 weeks prior to sacrifice $[17,18,46]$. Animals are then deeply anesthetized and transcardially perfused with $4 \%$ paraformaldehyde. Rostrocaudal lesion location is determined from the dorsal root entry zones. An 8-mm block centered on the lesion is removed, cryoprotected in $20 \%$ glycerin, frozen, and sectioned horizontally at $30-\mu \mathrm{m}$ intervals. Each twelfth section is stained for Nissl and used for lesion reconstruction. Immunohistochemistry and electron microscopy techniques are used for further careful anatomical analyses.

Using the surgical technique described herein, nearly all cases of complete hemisections are accomplished in the $\mathrm{C} 7$ segment or at the C7-8 junction (for more details see Rosenzweig et al. [18] and Nout et al. [43]). Many lesions encroach slightly on the contralateral side of the spinal cord, affecting the medial aspect of the dorsal funiculus, ventral funiculus, and/or gray matter.

\section{Statistical Procedures}

Investigators performing the functional testing tasks, data collection, and analysis are blind to group identities. Datasets from the electrophysiology, kinematics, chair tasks, open field testing, and anatomy are combined into 1 single large database. Data are analyzed using univariate and multivariate statistics, including principal component analysis [18].

\section{Contributions and Implications: Neuroanatomy and Plasticity}

Initial studies performed during development of this model were aimed at discerning unique characteristics of locomotion in the Rhesus monkey compared to other quadrupedal mammals [41], and at determining how a low thoracic corticospinal tract lesion would affect quadrupedal stepping in the monkey [42]. These studies were performed to create a primate model of SCI that produced persistent deficits in locomotion and fine motor control, and in which neural repair interventions could be tested. These studies provided baseline data for kinematics and EMG determinants in the monkey, and showed similarities and differences between the Rhesus monkey, sub-primate mammal, and human locomotion. After lesions of the thoracic dorsolateral column, monkeys showed initial loss of function, followed by substantial recovery [42]. Similar to prior reports, these studies 


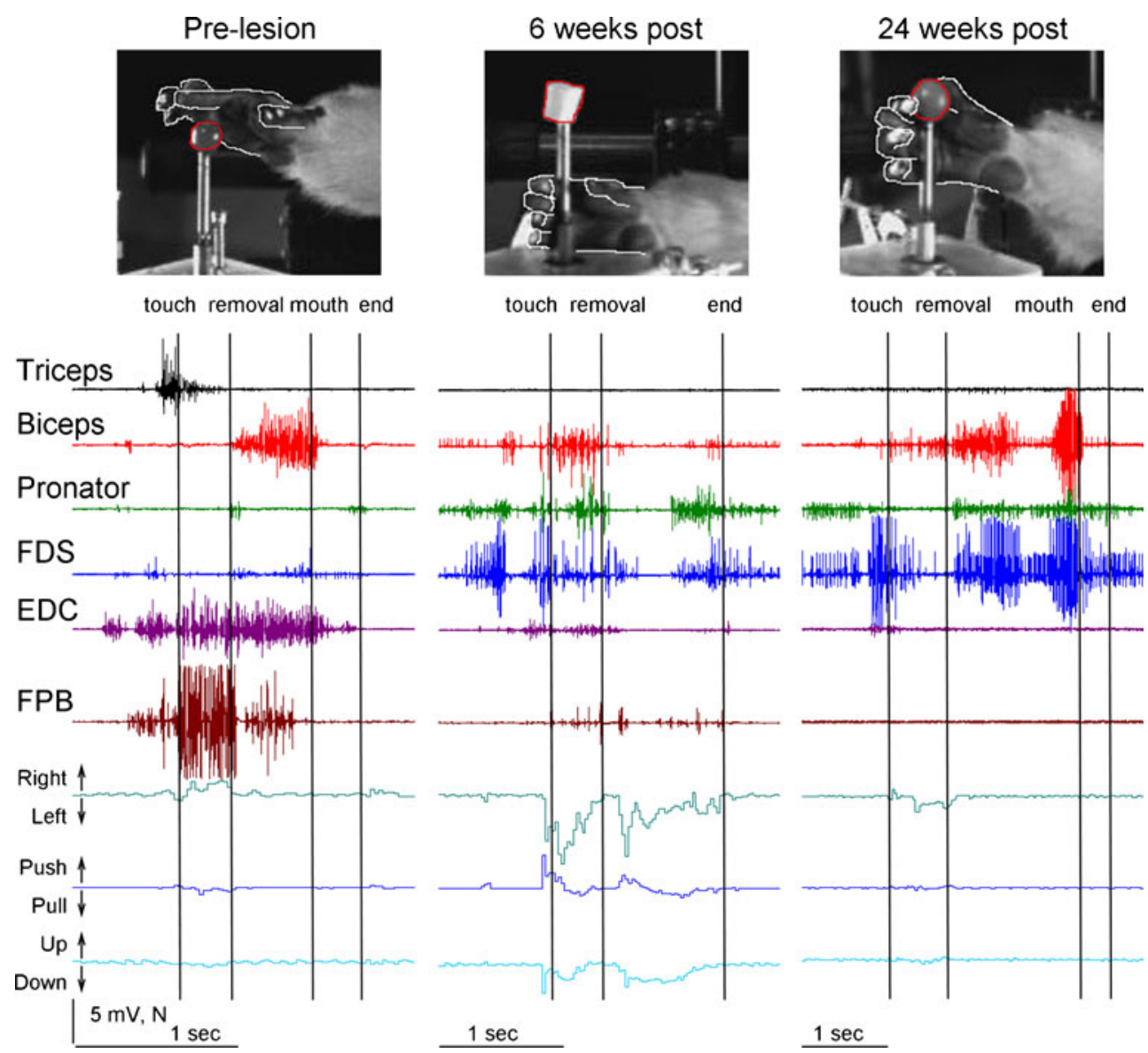

Fig. 2 Recovery of the "grape-on-the-stick" hand task. The top panel shows images taken of the hand while the monkey grasps a grape from a stick, and the panel below shows the corresponding electromyographic (EMG) tracings for 6 forelimb muscles during this task. A force plate sensor is located underneath the platform that holds the stick (on which the food is placed), and readings from this sensor are shown in the bottom 3 graphs. This hand task involves the monkey reaching out to touch the food (touch), then remove the food from the stick (removal), followed by bringing the food to the monkey's mouth (mouth). Pre-lesion, before spinal cord injury (SCI), the monkey uses a pincer grasp to grasp the grape from the stick. The item is then successfully brought to the monkey's mouth. Triceps, biceps, extensor digitorum communis (EDC), and flexor pollicis brevis (FPB) are all activated during this task, as seen by the EMG activity for these muscles. Pre-injury, retrieval of the food object from the stick occurs with little to no force applied to the stick/plate. At 6 weeks after SCI, this monkey regained the ability to touch and remove the food from the stick, however, not by using a pincer grasp. This monkey placed his hand around the bottom of the stick and lifted his arm, thereby removing the food item. It was then dropped and was thus not transported to

showed that the primary motor cortex plays an important role in controlling locomotion in the monkey, and significantly affects stepping-related spinal circuitries. In addition, the corticospinal tract arising from the contralateral primary motor cortex was found to be a main route through which volitional distal foot movements are controlled, similar to earlier results in the forelimb [13]. These studies showed that the corticospinal tract is normally involved in inter-limb the monkey's mouth. From the EMG it can be seen that some muscles that were active during this task before SCI (triceps, EDC, FPB) are not or only very slightly activated, whereas others that were not active before SCI (pronator, flexor digitorum superficialis [FDS]) are being activated after SCI. In addition, the biceps muscle that is used when food is brought to the mouth (before SCI) is now activated mainly when the animal is removing the item from the stick. After SCI, the animal is not able to retrieve the object in a clean controlled fashion, and forces (in the 3 measured dimensions) are applied to the stick and/ plate that are recorded by the force plate sensor. At 6 months after SCI, the animal is able to grasp the food item and bring it to its mouth. However, the grasping method is different from before SCI, and this is also reflected by the EMG patterns that display stronger activation of the pronator and FDS muscles with a more normal pattern seen in the biceps muscle. However, the triceps muscle, the EDC, and the FPB are not activated during this task. With time, the animal regains the ability to control hand movements and perform this task in a clean, controlled fashion, with little to no forces applied to the stick (i.e., similar to preinjury ability)

and intra-limb coordination, as well as in activation of specific muscles required for dexterous foot digit movements. Recovery of the more proximal musculature after SCI was better than recovery of distal musculature, and recovery of automated locomotor movements was better than recovery of tasks requiring fractionated control of distal digits [42]. These patterns may be attributable to the bilateral projection pattern of brainstem projections to intraspinal 
Hindlimb locomotion

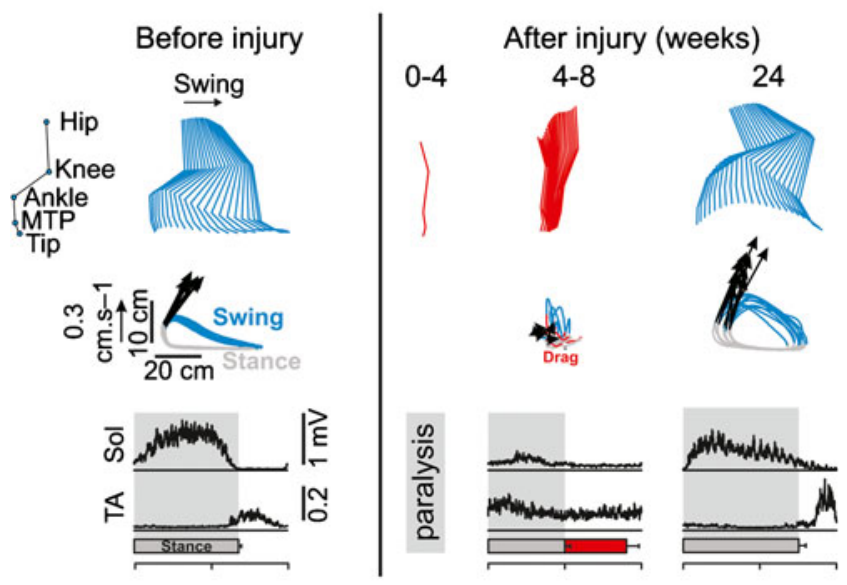

Forelimb locomotion

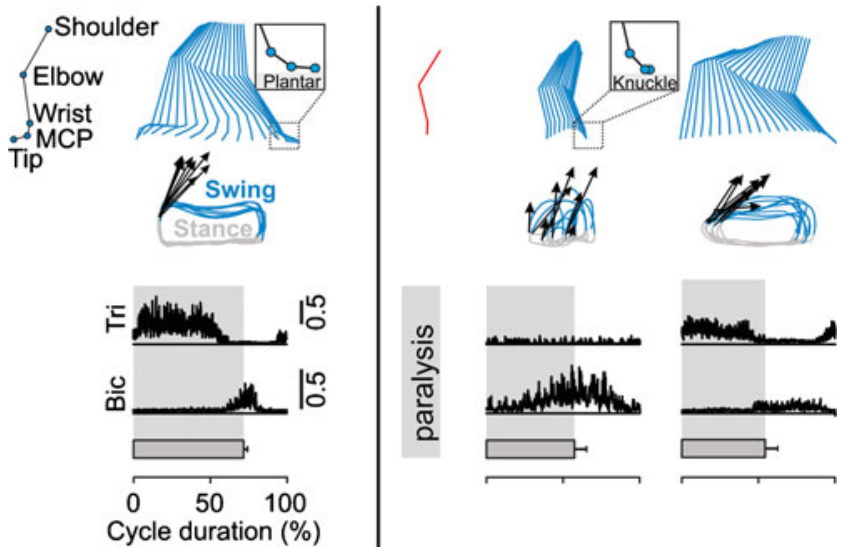

Fig. 3 Recovery of locomotion after spinal cord injury. Representative stick diagram decompositions (30 ms between sticks) of lesion side hindlimb and forelimb movements during the swing phase while stepping quadrupedally on the treadmill at $0.45 \mathrm{~ms}^{-1}$ before and at different time points postinjury. The successive ( $n=10$ steps), colorcoded trajectories (blue, swing; red, drag; gray, stance) of the hindlimb endpoint (metatarsophalangeal [MTP]) and forelimb endpoint (metacarpophalangeal $[\mathrm{MCP}]$ ) are shown together with the intensity and direction of the endpoint velocities (arrows) at swing onset. Mean integrated EMG activity ( $\mathrm{n}=10$ steps) of selected hindlimb (soleus [Sol]; tibialis anterior [TA]) and forelimb (triceps [Tri]; biceps [Bic]) muscles is shown at the bottom of the panel for each time point. Shaded areas indicate the duration of the stance phase and red bars indicate the duration of paw dragging

locomotor circuitry [47]. Nonetheless, the ability of the primate central nervous system to rapidly accommodate a reduction in supraspinal control of locomotion and, to a lesser degree, of fine motor skills of the foot, emphasizes the high degree of plasticity of the primate central nervous system. Because cervical injury is the most common site of SCI in humans, and mid-cervical lesions create a relatively short distance along which treated axons must grow to potentially influence functional outcomes, we now perform lesions at the $\mathrm{C} 7$ spinal cord level (corresponding to the C5-6 vertebral level).
The first significant observations to arise from our studies of the primate addressed the anatomy of intact cervical spinal cord corticospinal projections $[14,17]$. We found that large numbers of corticospinal tract axons branch and then decussate across the cervical spinal cord midline, resulting in bilateral terminations. These studies showed that $87 \%$ of corticospinal tract axons descend in the dorsolateral tract of the spinal cord contralateral to the hemisphere of origin, whereas $11 \%$ of axons descend in the dorsolateral fasciculus ipsilateral to the hemisphere of origin. Surprisingly, almost twice as many axons decussate across the cervical spinal cord midline, as those that descend in the dorsolateral funiculus of the spinal cord, suggesting that each corticospinal tract axon extends multiple segmental collaterals. Furthermore, reconstruction of individual corticospinal axons showed an unprecedented degree of bilaterality of terminal arbors [17]. In contrast, in rats, 96 to $98 \%$ of corticospinal tract projections decussate in the medullary pyramids, and axonal decussation across the spinal cord midline is uncommon (Fig. 4) [48]. These findings have significant implications for promoting neural plasticity after injury because the bilateral nature of the primate corticospinal tract and the presence of spinally decussating corticospinal tract pathways could be a potential target for therapeutic intervention after incomplete SCI. Indeed, the nearly uniform spontaneous improvement in function after incomplete human SCI likely arises, at least in part, from the plasticity of these midline-decussating corticospinal tract axons.

Then we began to study animals with $\mathrm{C} 7$ hemisection lesions. Following $\mathrm{C} 7$ hemisections, primates demonstrated a substantial degree of spontaneous plasticity of corticospinal tract axons that restored axon density in the gray matter below the lesion to $60 \%$ of normal levels [18], despite the removal of approximately $90 \%$ of the projecting axons. In addition, the degree of spontaneous plasticity was correlated with substantial improvements in both skilled hand and locomotor function using kinematics and EMG

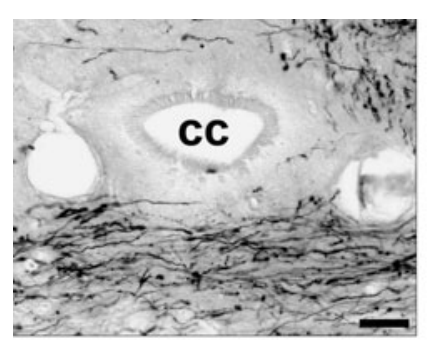

Primate

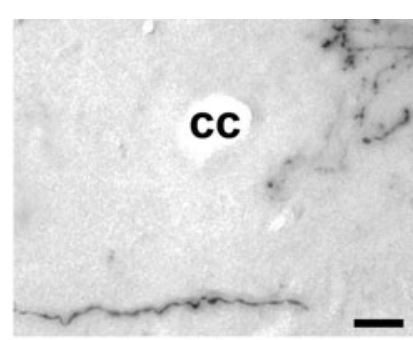

Rat
Fig. 4 Midline crossing corticospinal axons. Corticospinal axons labeled with biotinylated dextran amine, crossing spinal cord midline in Rhesus macaque (left) and rat (right). Note the large number of midline crossing corticospinal tract axons in the primate spinal cord, and the rarity of such axons in the rat. $\mathrm{cc}=$ central canal. Scale bars: $50 \mu \mathrm{m}$ (primate), $25 \mu$ (rat) 
parameters defined previously for these tasks in uninjured animals $[41,45]$. These findings not only identified extensive natural recovery after nervous system injury in the primate, but they also highlighted the importance of this primate model for translational disease research because these findings differ from observations in rodents. In rat models of SCI (dorsal column lesions and hemisections) only 3 to $5 \%$ of pre-injury axon density is reconstituted after cervical hemisection $[49,50]$, which is far less than the $60 \%$ observed in the primate. Because corticospinal axons extensively decussate across the spinal cord midline in primates $[14,17]$, the primate retains a larger pool of supraspinal influence below a lateral hemisection than rodents. This extensive spontaneous increase in corticospinal axon density may in fact be the mechanism that accounts for the marked improvement in function seen in humans after incomplete SCI. Similar to the approximately $50 \%$ recovery of hand function observed in this nonhuman primate model of SCI, humans recover locomotor function and partial hand function after Brown-Sequard lesions [51]. In contrast, rats do not recover the ability to grasp food after a SCI hemisection [52]. Although our research is focused on the involvement of the corticospinal system in recovery of function, evaluation of other systems and tracts involved in motor function, such as the propriospinal system, will be pursued in future studies.

In addition to studying the uninjured primate central nervous system and spontaneous responses to injury, we have used this nonhuman primate model of SCI to study responses to promising regenerative therapeutic interventions derived from rodent models of SCI, including cellular transplantation strategies $[23,26]$ and growth factor gene delivery $[24,25]$. After transplantation of genetically modified fibroblasts that secrete brain-derived neurotrophic factor (BDNF) and neutrotrophin-3 (NT-3) into the lesion site, plus local spinal cord injections of lentivirus encoding BDNF or NT-3, we found long distance effects of growth factors in the primate central nervous system [46]. Specifically, growth factors applied at the lesion site prevented axotomy-induced atrophy of corticospinal cell bodies in the motor cortex, approximately $10-\mathrm{cm}$ distant. Moreover, growth factors expressed by cell grafts placed in the lesion site recruited the growth of several axonal populations into the lesion site, however, such growth did not include corticospinal systems, [46]. These experiments are continuing.

\section{Contributions and Implications: Method Development for Therapeutic Delivery}

In a more recent study in which candidate therapies were injected directly into the spinal cord after a complete lateral hemisection, we discovered an unexpected and dramatic loss of spinal cord neurons near injection sites of all substances except for saline [27]. This neuronal loss was not accompanied by spinal cord cavitation or obvious parenchymal injury at the injection site. In addition, animals that received injections had more severe motor deficits than noninjected (lesion-only) controls. Principal component analysis revealed a strong relationship between the degree of neuronal loss and impairment of forelimb function. It is important to recognize that injections of these same substances, using identical methods, did not cause detectable neuronal loss in rodents. Although injections of small volumes of drugs and solutes can be made into the central nervous system without obvious tissue damage, therapeutic spinal cord injections may carry additional challenges [53-55]. Often there is pre-existing tissue damage, delivery volumes may be large, and cell or solute concentrations are high. In addition, delivery rates may be high to complete these treatments within the realistic time parameters of a human operative procedure. Injection injury likely occurs through mechanisms similar to traumatic injury, such as compression, laceration, excitotoxicity, apoptosis, and ischemia. Also, pressure-based injections of larger volumes may lead to damage through elevated intraparenchymal pressures that consequently result in hydrodynamic tissue dissection and possible ischemia [53]. The finding that injections into the primate spinal cord cause cell loss that is not observed in rodent models highlights the need for preclinical testing of therapies in primates $[53,55]$. It is better to discover negative side effects from therapies or techniques in an animal model rather than having human clinical trials fail for unknown reasons. Postmortem examinations are often unavailable in humans or not available for long periods of time. We are currently investigating mechanisms underlying the unexpected loss of motor neurons in these primate experiments to facilitate the design and implementation of methods for safe therapeutic delivery in future studies.

Recovery from SCI is largely dependent on the amount of spared tissue at the level of the lesion center. Therefore, it is critical that preserved axons and neurons should not undergo further damage by procedures that are aimed at repair. Further research is required in the field of central nervous system drug delivery methods. For example, it may be possible to prevent the "pistoning effect" (spinal cord cavity formation secondary to combined rostral-caudal and ventraldorsal motion when a needle is fixed in place) with either percutaneous endoscopy and placement of a needle into the spinal cord without it being held fixed in place [53], or with a needle that sits on the cord surface and moves with the cord [56]. Although local injection techniques have the advantage of precise delivery of injectate to the target, less invasive techniques, such as use of intrathecal injections or scaffolds, could be considered for treatment delivery. Scaffolds not only provide guidance and orientation of axons 
after injury $[57,58]$, but extended release scaffolds also can be loaded with substances [59] and should be considered as a less invasive method for intraparenchymal drug delivery.

\section{Contributions and Implications: Safety Assessment}

Assessing safety of experimental therapeutics is very important. During the course of our studies, we examine animals for adverse effects, including systemic illness, weight loss, depression, anorexia, vomiting, and diarrhea. In addition, we routinely assess signs of abnormal behavior, including aggression, tremor, spasticity, or self-injury. Finally, all animals undergo complete necropsy and central nervous system histopathology to identify any organ abnormalities or neoplasia. So far, the most dramatic adverse effect we have identified is motor neuron loss associated with spinal cord injections [27], as previously discussed. We have identified the development of spasticity in some animals [43] and are developing physiotherapeutic exercises to prevent animals from developing joint rigidity and nonuse. In addition, we are currently exploring methods to assess pain in our model.

Because humans with SCI often report allodynia or chronic neuropathic or central pain, we have been careful to assess animals for signs of distress or discomfort. Although monkeys in our studies have not exhibited overt signs of chronic pain (e.g., reduced appetite, inactivity, or vocalizations), we have seen signs consistent with altered sensation. In humans, dysesthesias are commonly found in body parts in which there is reduced or no sensory response to peripheral somatic stimulation (e.g., in hypoesthesia, hypoalgesia, anesthesia, analgesia). Earlier studies in monkeys have shown that monkeys can appear to develop hyperesthesia at the border of the sensory level after chronic spinal cord transections [60], and occasional abnormal, compulsive, self-directed behaviors have been reported after thoracic spinal cord lesions [61, 62]. The studies of other investigators report that animals displayed compulsive rubbing, depilation, and biting of areas within the hypoalgesic dermatomes after mid-thoracic anterolateral cordotomy or hemisection. Similar signs in rodent SCI have sometimes been reported, and it is not clear whether these behaviors are directed at a site of discomfort or at a body part that is insensate, and therefore may be identified as foreign by the animal [63]. Note that these clinical signs occur on the side of the animal that does not display motor deficits. Levitt [64] concluded that the "deafferentation syndrome" was released by interrupting spinothalamic tracts, and that the incidence was genetically variable (incidence of $31 \%$ in a multispecies group to $95 \%$ in a group of 19 stump-tailed macaques), and that some of the variability might be related to variability in the location of the spinothalamic tracts (sometimes in the posterolateral funiculus and sometimes in the ipsilateral anterolateral funiculus) [61, 64]. In those earlier studies, monkeys that had sensorimotor deficits did not display disruptions of activity level, feeding, grooming (normal dermatomes), libido, vocalization, or social behavior. It is not known what level of altered sensation or discomfort is associated with these dysesthesias and how they might compare to the pain syndromes sometimes occurring after SCI in humans.

In our C7 hemisection model, most animals engage in chronic scratching and picking along the dorsal aspect of the neck, and scratching or picking of the contralateral (left) hindlimb resulting in superficial abrasions on the affected limb $(\sim 80 \%)$. Similar to the studies previously described, however, we do not see evidence of clinical pain associated with these behaviors. A few subjects ( 4 of 71 ) have required early euthanasia due to self-injurious behavior to the contralateral limb that was not readily stopped with gabapentin and neuroleptic treatment. Behavioral testing is complex in animals that are bandaged or are treated with drugs that alter cognitive performance. The effect of these therapeutic agents on recovery of function has yet to be determined.

Because changes in sensory function are important to take into consideration when exploring novel treatment interventions, we are in the process of expanding our sensory assessment techniques. We are adding sensory testing for mechanical allodynia and cold hyperalgesia using von Frey filaments and a cold probe in dermatomes above, at, and below the lesion. In addition, we are investigating the use of telemetric monitoring of blood pressure and heart rate with implantable pressure transducers as an autonomic measure and to detect distress, which can produce changes in heart rate and blood pressure [65]. Because our program is aimed at translational research, it is important to assess sensation in a manner that is relevant to humans. For example, humans are able to rate ongoing pain from applied stimuli. Although stimulus response functions for escape from nociceptive stimuli can provide some comparable information from laboratory animals [66], assessment of "pain" requires reporting of the affective state associated with the altered sensation, and this has not been easy to accomplish in animals, including nonhuman primates. Recently, paradigms for assessing the aversive qualities of ongoing "painful" sensation have been reported for rodents [67], and these may be adapted to nonhuman primates after C7 hemisection to monitor the effects of therapies on recovery. Such an approach also could be used to evaluate whether current pharmacological strategies aimed at alleviating discomfort after SCI in nonhuman primates (e.g., the administration of gabapentin in response to skin lesions contralateral to the lesion) are actually affecting aversive sensation, yielding information that might be translated to the clinical arena. Our goal is to devise methods to measure the presence and intensity of sensory changes in our SCI model that might be 
affected by therapeutic interventions. Any therapy that aims to unleash axonal growth to achieve a beneficial functional outcome has an accompanying risk of increasing nociceptive axon growth that would exacerbate pain. Accordingly, pain assessment is very important.

\section{Conclusions}

Primate studies should be undertaken judiciously. Some therapies for SCI are likely to require interventions with moderate to high risk, including cellular transplantation and surgical manipulations that may be quite different in rodents and larger nonhuman primates. Furthermore, recovery of hand and digit function is especially important after cervical SCI in humans, and although similarities in rodent and primate recovery are seen, it is quite clear there are significant differences, especially with respect to the corticospinal tract [18]. Our primate model is intended to: 1) lend greater positive predictive value to human translatable therapies; 2) allow appropriate methods to be developed for human translation, improving the potential for successful human trials; 3) lead to basic discoveries that might not be identified in rodent models and are relevant to human translation; and 4) identify new avenues of basic research to "reverse-translate" important questions back to rodent models. We have demonstrated significant differences between rodents and primates in both intact neuroanatomy and neuroanatomical responses to SCI. In addition, we have shown the importance of testing therapeutic techniques in nonhuman primates prior to using aggressive surgical techniques in human clinical trials [27].

Now we have a nonhuman primate model of SCI in which detailed functional and anatomical outcomes can be measured to investigate the effects of regenerative therapeutic treatment strategies. Studies with this model can be considered "nonhuman primate clinical trials" as they present an opportunity to discover, with the added benefit of postmortem examinations, adverse effects or negative findings in a model closer to humans than the rodent model.

We are continuing to develop this model to ensure that it is used to its fullest extent and to enhance its clinical relevance. For example, the recent addition of the openfield behavioral scoring has allowed evaluation of spontaneous and naturalistic behaviors. Ongoing studies are focused on developing a partial contusion injury. In addition, studies are underway to include assessment of sensation and autonomic function in this model. Finally, the interaction of rehabilitation paradigms with therapeutics to enhance axonal growth is a topic of substantial potential importance, and is being actively investigated. We note that the present model, a C7 hemisection, is similar in nature to a human
Brown-Sequard injury that accounts for only $3 \%$ of all clinical cases of SCI [68]. Thus, although this model is useful in assessing therapies that target new axonal growth (sprouting or regeneration), it is currently less relevant than contusive rodent models for assessing neuroprotective strategies to improve outcomes after SCI. Finally, the purchase, housing and detailed training of monkeys are more costly than rodent studies by a factor of perhaps 5 to 10 -fold. Thus, the potential benefits and limitations of the model must be judiciously considered using the primate resource to advance SCI research. Ultimately, the success of the multidimensional effort of this consortium will be judged by its impact on the development of therapies for humans.

Acknowledgments This work was supported by the United States National Institutes of Health (NS42291, NS049881, and NS067092), the Veterans Administration, California Roman-Reed funds, and Bernard and Anne Spitzer Charitable Trust.

Required Author Forms Disclosure forms provided by the authors are available with the online version of this article.

\section{References}

1. Blight AR and Tuszynski MH. Clinical trials in spinal cord injury. J Neurotrauma 2006;23:586-593.

2. Courtine G, Bunge MB, Fawcett JW, et al. Can experiments in nonhuman primates expedite the translation of treatments for spinal cord injury in humans? Nat Med 2007;13:561-566.

3. Kwon BK, Casha S, Hurlbert RJ, et al. Inflammatory and structural biomarkers in acute traumatic spinal cord injury. Clin Chem Lab Med 2011;49:425-433.

4. DeVivo MJ, Chen Y. Trends in new injuries, prevalent cases, and aging with spinal cord injury. Arch Phys Med Rehabil 2011;92:332-338.

5. Anderson KD. Targeting recovery: priorities of the spinal cordinjured population. J Neurotrauma 2004;21:1371-1383.

6. National Spinal Cord Injury Statistical Center. 2011 Annual Report for the Spinal Cord Injury Model Systems. University of Alabama. https://www.nscisc.uab.edu/PublicDocuments/reports/pdf/ $2010 \% 20$ NSCISC $\% 20$ Annual $\% 20$ Statistical $\% 20$ Report $\% 20$ $\% 20$ Complete $\% 20$ Public $\% 20$ Version.pdf.

7. Gensel JC, Tovar CA, Hamers FP, et al. Behavioral and histological characterization of unilateral cervical spinal cord contusion injury in rats. J Neurotrauma 2006;23:36-54.

8. Tuszynski MH, Grill R, Jones LL, et al. Spontaneous and augmented growth of axons in the primate spinal cord: effects of local injury and nerve growth factor-secreting cell grafts. J Comp Neurol 2002;449:88-101.

9. Edgerton VR, Roy RR. Paralysis recovery in humans and model systems. Curr Opin Neurobiol 2002;12:658-667.

10. Eidelberg E, Walden JG, Nguyen LH. Locomotor control in macaque monkeys. Brain 1981;104:647-663.

11. Vilensky JA, O'Connor BL. Stepping in nonhuman primates with a complete spinal cord transection: old and new data, and implications for humans. Ann N Y Acad Sci 1998;860:528-530.

12. Galea MP, Darian-Smith I. Corticospinal projection patterns following unilateral section of the cervical spinal cord in the newborn and juvenile macaque monkey. J Comp Neurol 1997;381:282-306. 
13. Lawrence DG, Kuypers HG. The functional organization of the motor system in the monkey. I. The effects of bilateral pyramidal lesions. Brain 1968;91:1-14.

14. Lacroix S, Havton LA, McKay H, et al. Bilateral corticospinal projections arise from each motor cortex in the macaque monkey: a quantitative study. J Comp Neurol 2004;473:147-161.

15. Lemon RN, Griffiths J. Comparing the function of the corticospinal system in different species: organizational differences for motor specialization? Muscle Nerve 2005;32:261-279.

16. Nathan PW, Smith MC. The rubrospinal and central tegmental tracts in man. Brain 1982;105:223-269.

17. Rosenzweig ES, Brock JH, Culbertson MD, et al. Extensive spinal decussation and bilateral termination of cervical corticospinal projections in rhesus monkeys. J Comp Neurol 2009;513:151163.

18. Rosenzweig ES, Courtine G, Jindrich DL, et al. Extensive spontaneous plasticity of corticospinal projections after primate spinal cord injury. Nat Neurosci 2010;13:1505-1510.

19. Blesch A, Tuszynski MH. Cellular GDNF delivery promotes growth of motor and dorsal column sensory axons after partial and complete spinal cord transections and induces remyelination. J Comp Neurol 2003;467:403-417.

20. Grill R, Murai K, Blesch A, et al. Cellular delivery of neurotrophin-3 promotes corticospinal axonal growth and partial functional recovery after spinal cord injury. J Neurosci 1997;17:5560-5572.

21. Jones LL, Sajed D, Tuszynski MH. Axonal regeneration through regions of chondroitin sulfate proteoglycan deposition after spinal cord injury: a balance of permissiveness and inhibition. J Neurosci 2003;23:9276-9288.

22. Lu P, Jones LL, Snyder EY, et al. Neural stem cells constitutively secrete neurotrophic factors and promote extensive host axonal growth after spinal cord injury. Exp Neurol 2003;181:115-129.

23. Lu P, Jones LL, and Tuszynski MH. BDNF-expressing marrow stromal cells support extensive axonal growth at sites of spinal cord injury. Exp Neurol 2005;191:344-360.

24. Lu P, Yang H, Jones LL, et al. Combinatorial therapy with neurotrophins and cAMP promotes axonal regeneration beyond sites of spinal cord injury. J Neurosci 2004;24:6402-6409.

25. Tuszynski MH, Gabriel K, Gage FH, et al. Nerve growth factor delivery by gene transfer induces differential outgrowth of sensory, motor, and noradrenergic neurites after adult spinal cord injury. Exp Neurol 1996;137:157-173.

26. Tuszynski MH, Peterson DA, Ray J, et al. Fibroblasts genetically modified to produce nerve growth factor induce robust neuritic ingrowth after grafting to the spinal cord. Exp Neurol 1994;126:1-14.

27. Rosenzweig ES, Courtine G, Brock JH, et al. Unexpected neuronal loss after spinal cord injections in primates. In: Society for Neuroscience 2011:255.08/Z25.

28. Mettler FA, Liss H. Functional recovery in primates after large subtotal spinal cord lesions. J Neuropathol Exp Neurol 1959;18:509516.

29. Turner WA. On hemisection of the spinal cord. Brain 1891;14:496522.

30. Denny-Brown D. The cerebral control of movement. Liverpool, UK, 1966.

31. Mettler FA. Observations on the consequences of large, subtotal lesions of the simian spinal cord. J Comp Neurol 1944;81:339-360.

32. Brinkman J, Bush BM, Porter R. Deficient influence of peripheral stimuli on precentral neurones in monkeys with dorsal column lesions. J Physiol 1978;276:27-48.

33. Glendinning DS, Vierck CJ Jr., Cooper BY. The effect of fasciculus cuneatus lesions on finger positioning and long-latency reflexes in monkeys. Exp Brain Res 1993;93:104-116.

34. Vierck CJ Jr., Hamilton DM, Thornby JI. Pain reactivity of monkeys after lesions to the dorsal and lateral columns of the spinal cord. Exp Brain Res 1971;13:140-158.
35. Vierck CJ Jr., Luck MM. Loss and recovery of reactivity to noxious stimuli in monkeys with primary spinothalamic cordotomies, followed by secondary and tertiary lesions of other cord sectors. Brain 1979;102:233-248.

36. Tower S. Pyramidal lesions in the monkey. Brain 1940;63:36-90.

37. Galea MP, Darian-Smith I. Manual dexterity and corticospinal connectivity following unilateral section of the cervical spinal cord in the macaque monkey. J Comp Neurol 1997;381:307-319.

38. Hepp R, Trouche E, Wiesendanger M. Effects of unilateral and bilateral pyramidotomy on a conditioned rapid precision grip in monkeys (Macaca fascicularis). Exp Brain Res 1974;21:519-527.

39. Sasaki S, Isa T, Pettersson LG, et al. Dexterous finger movements in primate without monosynaptic corticomotoneuronal excitation. J Neurophysiol 2004;92:3142-3147.

40. Rouiller EM, Yu XH, Moret V, et al. Dexterity in adult monkeys following early lesion of the motor cortical hand area: the role of cortex adjacent to the lesion. Eur J Neurosci 1998;10:729-740.

41. Courtine G, Roy RR, Hodgson J, et al. Kinematic and EMG determinants in quadrupedal locomotion of a nonhuman primate (Rhesus). J Neurophysiol 2005;93:3127-3145.

42. Courtine G, Roy RR, Raven J, et al. Performance of locomotion and foot grasping following a unilateral thoracic corticospinal tract lesion in monkeys (Macaca mulatta). Brain 2005;128:2338-2358.

43. Nout YS, Ferguson AR, Strand SC, et al. Methods for functional assessment after $\mathrm{C} 7$ spinal cord hemisection in the rhesus monkey. Neurorehabilitation and neural repair. 2012. doi:10.1177/ 1545968311421934.

44. Bohannon RW, Smith MB. Interrater reliability of a modified Ashworth scale of muscle spasticity. Phys Ther 1987;67:206-207.

45. Jindrich DL, Courtine G, Liu JJ, et al. Unconstrained threedimensional reaching in Rhesus monkeys. Exp Brain Res 2011;209:35-50.

46. Brock JH, Rosenzweig ES, Blesch A, et al. Local and remote growth factor effects after primate spinal cord injury. J Neurosci 2010;30:9728-9737.

47. Kuypers H. Anatomy of the descending pathways. In: Handbook of physiology. Brooks V, Brookhart J, VBM, eds. The American Physiological Society, Bethesda, MD. 1981, pp. 597-666.

48. Rouiller EM, Liang FY, Moret V, et al. Trajectory of redirected corticospinal axons after unilateral lesion of the sensorimotor cortex in neonatal rat; a phaseolus vulgaris-leucoagglutinin (PHA-L) tracing study. Exp Neurol 1991;114:53-65.

49. Weidner N, Ner A, Salimi N, et al. Spontaneous corticospinal axonal plasticity and functional recovery after adult central nervous system injury. Proc Natl Acad Sci U S A 2001;98:3513-3518.

50. Ghosh A, Sydekum E, Haiss F, et al. Functional and anatomical reorganization of the sensory-motor cortex after incomplete spinal cord injury in adult rats. J Neurosci 2009;29:12210-12219.

51. Roth EJ, Park T, Pang T, et al. Traumatic cervical Brown-Sequard and Brown-Sequard-plus syndromes: the spectrum of presentations and outcomes. Paraplegia 1991;29:582-589.

52. Anderson KD, Gunawan A, Steward O. Quantitative assessment of forelimb motor function after cervical spinal cord injury in rats: relationship to the corticospinal tract. Exp Neurol 2005;194:161-174.

53. Guest J, Benavides F, Padgett K, et al. Technical aspects of spinal cord injections for cell transplantation. Clinical and translational considerations. Brain Res Bull 2011;84:267-279.

54. Usvald D, Vodicka P, Hlucilova J, et al. Analysis of dosing regimen and reproducibility of intraspinal grafting of human spinal stem cells in immunosuppressed minipigs. Cell Transplant 2010;19:1103-1122.

55. Riley J, Federici T, Park J, et al. Cervical spinal cord therapeutics delivery: preclinical safety validation of a stabilized microinjection platform. Neurosurgery 2009;65:754-762.

56. Riley JP, Raore B, Taub JS, et al. Platform and Cannula Design Improvements for Spinal Cord Therapeutics Delivery. Neurosurgery 2011;69(2 Suppl Operative):ons147-54; discussion ons155. 
57. Gros T, Sakamoto JS, Blesch A, et al. Regeneration of long-tract axons through sites of spinal cord injury using templated agarose scaffolds. Biomaterials 2010;31:6719-6729.

58. Zhu Y, Wang A, Patel S, et al. Engineering bi-layer nanofibrous conduits for peripheral nerve regeneration. Tissue Eng Part C Methods 2011;17:705-715.

59. Zhu Y, Wang A, Shen W, et al. Nanofibrous patches for spinal cord regeneration. Advanced Functional Materials 2010;20:14331440.

60. Denny-Brown D, Kirk EJ, Yanagisawa N. The tract of Lissauer in relation to sensory transmission in the dorsal horn of spinal cord in the macaque monkey. J Comp Neurol 1973;151:175-200.

61. Levitt M, Levitt JH. The deafferentation syndrome in monkeys: dysesthesias of spinal origin. Pain 1981;10:129-147.

62. Vierck CJ Jr., Greenspan JD, Ritz LA. Long-term changes in purposive and reflexive responses to nociceptive stimulation following anterolateral chordotomy. J Neurosci 1990;10:2077-2095.
63. Christensen MD, Everhart AW, Pickelman JT, et al. Mechanical and thermal allodynia in chronic central pain following spinal cord injury. Pain 1996;68:97-107.

64. Levitt M. Dysesthesias and self-mutilation in humans and subhumans: a review of clinical and experimental studies. Brain Res Reviews 1985;10:247-290.

65. Carrier CA, Elliott TB, Ledney GD. Real-time telemetric monitoring in whole-body 60Co gamma-photon irradiated rhesus macaques (Macaca mulatta). J Med Primatol 2010;39:399-407.

66. Vierck CJ, Hansson PT, Yezierski RP. Clinical and pre-clinical pain assessment: are we measuring the same thing? Pain 2008;135:710.

67. King T, Vera-Portocarrero L, Gutierrez T, et al. Unmasking the tonicaversive state in neuropathic pain. Nat Neurosci 2009;12:1364-1366.

68. McKinley W, Santos K, Meade M, et al. Incidence and outcomes of spinal cord injury clinical syndromes. J Spinal Cord Med 2007;30:215-224. 